Int. J. Dev. Biol. 50: 47-54 (2006)

doi: $10.1387 / \mathrm{ijdb} .052111 \mathrm{dh}$

Original Article

\title{
Comparative expression analysis of Pax3 and Pax7 during mouse myogenesis
}

\author{
DAVID HORST ${ }^{1,4}$, \#, SVETLANA USTANINA ${ }^{2}$, CONSOLATO SERGI ${ }^{3}$, GREGOR MIKUZ ${ }^{3}$, HERBERT JUERGENS ${ }^{1}$, \\ THOMAS BRAUN ${ }^{2}$ and EUGENE VOROBYOV ${ }^{4, *}$ \\ ${ }^{1}$ Klinik und Poliklinik für pädiatrische Hämatologie/Onkologie, Universitätsklinikum Münster, Germany, ${ }^{2}$ Max Planck Institut für Herz- und \\ Lungenforschung, Bad Nauheim, Germany, ${ }^{3}$ Institut für Pathologie, Medizinische Universität Innsbruck, Austria and ${ }^{4}$ Institut für \\ Humangenetik, Universitätsklinikum Münster, Germany
}

\begin{abstract}
Pax3 and Pax7 are closely related transcription factors involved in the commitment of myogenic precursors in the developing trunk. However, it is not yet clear whether these genes are required for myogenic cell specification in the head and for post-somitic myogensis per se. In part, this uncertainty is due to the scarce information about their normal time course and pattern of expression. Here, we present a systematic immunohistochemical in situ analysis of spatiotemporal characteristics of Pax3 and Pax7 protein expression in comparison to that of MyoD and myogenin in the developing trunk and head muscles. The observed patterns of expression suggest that Pax 3 is not involved in myogenesis in the head and its post-somitic expression in the trunk and limb muscles is mostly repressed after stage E13.5. In contrast, Pax7 expression is shared among all striated muscles and exhibits a uniform pattern. Pax 7 is expressed only in mononucleated cells that either differentiate into myotubes or later form satellite cells. During development of head muscles, expression of Pax 7 follows expression of $M y o D$ and myogenin, implying that Pax 7 is not required to induce the initial steps of the myogenic program in the head. In Pax7 homozygous mutants, in which muscle development proceeds normally, expression of Pax 3 is indistinguishable from its wild-type pattern (i. e. absent), suggesting that after stage E13.5 myogenesis does not require Pax 3 and Pax7. These data challenge the concept that Pax 3 and Pax 7 determine a persistent lineage of myogenic precursors in pre-natal and post-natal muscle development.
\end{abstract}

KEY WORDS: Pax3, Pax7, myogenesis, development

\section{Introduction}

Myogenesis is a multi-step process consisting of the determination of mesodermal precursor cells, myoblast migration and proliferation, cell fusion and terminal differentiation of myotubes. In vertebrates, all these steps are governed by sequential and compound activation of four myogenic regulatory factors (MRFs) that belong to the MyoD gene family: Myf5, MyoD, Mrf4 and myogenin (see Arnold and Braun, 1996). Functions of these genes have been defined by analyses of their single, double and triple mutants in mice. Recent data show that Myf5and Mrf4 play partially non-redundant roles in the determination of muscle identity and act upstream of MyoD (Kassar-Duchossoy et al., 2004). In turn, function of $M y O D$ significantly overlaps with functions of Myf5and Mrf4. Double mutants of MyoDwith either Myf5 or Mrf4 are characterized by an absence of skeletal muscles at birth (Rudnicki et al., 1993; Kassar-Duchossoy et al., 2004). In mice lacking myogenin, myoblasts are specified and positioned correctly, but their fusion capacity is greatly impaired. This indicates that myogenin is critical for terminal differentiation of myoblasts (Hasty et al., 1993; Nabeshima et al., 1993). Analysis of Myf5/ MyoDdouble mutants with an intact Mrf4gene revealed that skeletal myogenesis was absent in the head and, at later stages, severely compromised in the trunk, thus excluding a role for Mrf4 in the development of head muscles (Kassar-Duchossoy et al., 2004). Apparently, Myf5, MyoD and myogenin are essential activators of the myogenic program that are required in virtually all striated muscles. In contrast to the well established role of MRFs for muscle development, much less is known about genes which have been proposed to act upstream of MRFs to determine early

Abbreviations used in this paper: MRF, myogenic regulatory factor.

\footnotetext{
*Address correspondence to: Dr. Eugene Vorobyov. Institut für Humangenetik, Universitätsklinikum, Vesaliusweg 12-14, 48149 Münster, Germany. Fax: +49-251-83-56995. e-mail: vorobie@uni-muenster.de
} 


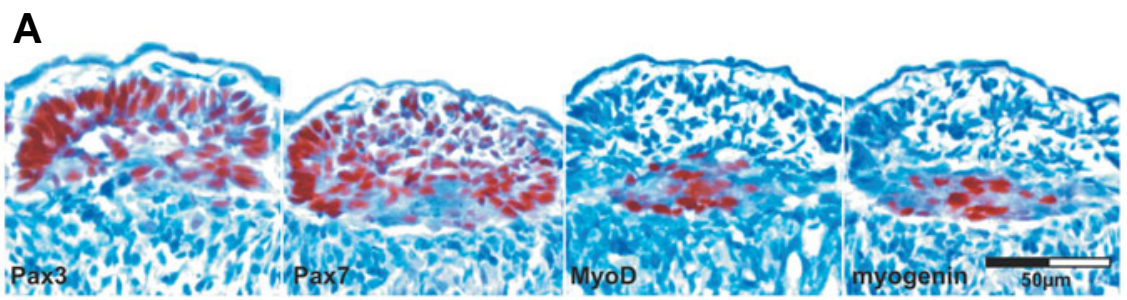

B

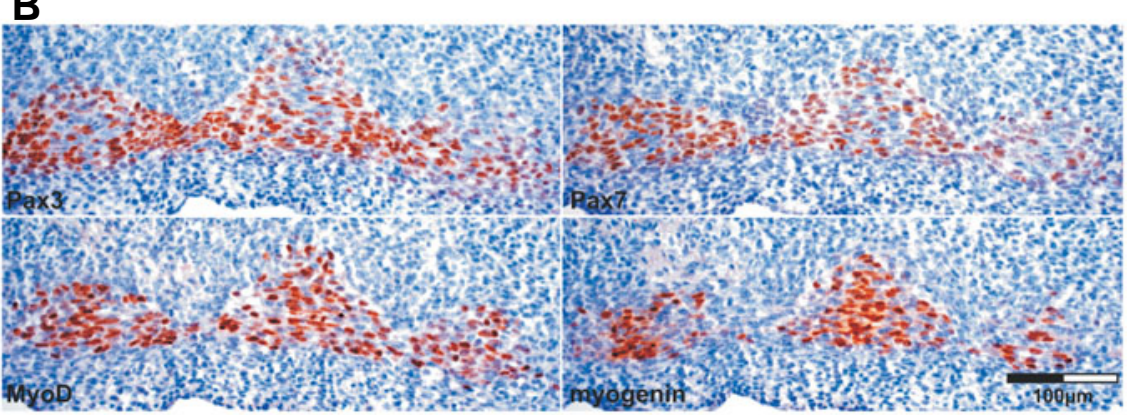

C

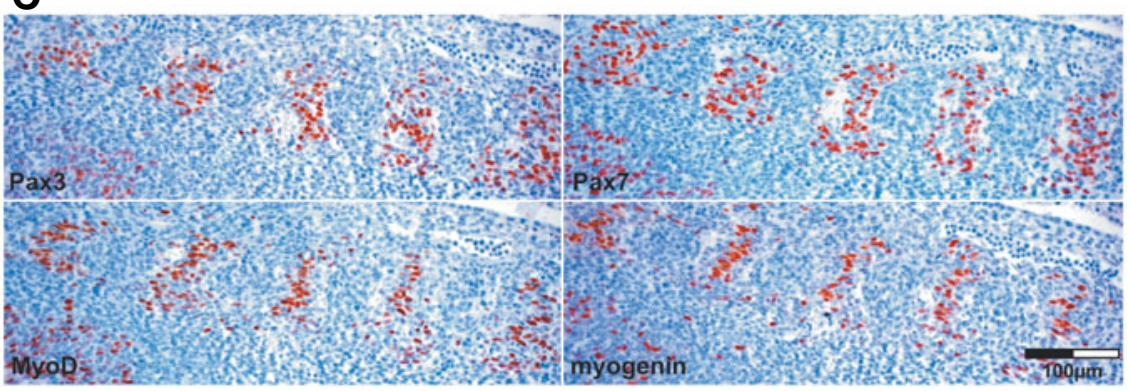

Fig. 1. Expression of Pax3, Pax7, MyoD and myogenin in somites. (A) The dermatome and myotome at E11.5 and the myotome at E11.5 (B) and E12.5 (C). The location of sections is shown in Figure 9.

steps of myogenic lineage commitment.

Two members of the Paxfamily of transcriptional factors, Pax3 and Pax7, are supposed to be involved in the myogenic cell specification. Both genes are known as early markers of the forming dermomyotome, which is the source of future myoblasts (Goulding et al., 1991; Jostes et al., 1990). The spontaneous Pax3 mutation, Splotch, when homozygous, is associated with the inability of myogenic precursors to migrate from somites into the growing limbs and the developing tongue and results in the complete absence of the respective muscles (Bober et al., 1994; Goulding et al., 1994; Tremblay et al., 1998). Homozygous Pax7 knockout mice develop normally but the newborns show a slightly reduced muscle mass and most mutants survive only a few weeks (Mansouri et al., 1996; Oustanina et al., 2004). Analysis of the affected muscles revealed a huge decrease of the number of satellite cells after birth indicating a role for Pax 7 in satellite cell self-renewal (Seale et al., 2000; Oustanina et al., 2004). Double knockouts of Pax3and Pax 7resulted in a compromised development of somites and embryonic lethality at mid-gestation, suggesting a redundancy in functions of both genes during somitogenesis. In these compound mutant mice myotomal myogenesis is initiated but resident muscle progenitor cells do not follow the myogenic program (Relaix et al., 2005). Evidence for a potential involvement of $P a x 3$ in myogenesis was also demonstrated by analysis of compound mutants of Pax3and Myf5 (Tajbakhsh etal., 1997). In the double homozygous embryos, skeletal muscle development in the trunk was missing, although the head muscles were essentially unaffected. The latter finding might be explained by a compensative role of Pax 7 in the head myogenesis, although this possibility was excluded in the same study since expression of both genes in the head mesoderm was not detected at stage E10.5. In chicken embryos, however, $P a x 7$ is expressed in at least one developing head muscle, the lateral rectus (Mootoosamy and Dietrich, 2002). In this study, the rudiment of the largest extrinsic eye muscle was found to co-express Pax 7 and an early somitic mesoderm marker Paraxis suggesting that in the cranial paraxial mesoderm Pax7may work upstream of MRFs and substitute for Pax3.

Currently, it is not clear whether and to what extent Pax3 and/or Pax 7 contribute to the progression of the myogenic process or whether they are involved in the development of head muscles. Skeletal muscles in the head and trunk are distinct in their origin. Most craniofacial muscles, except the tongue and laryngeal muscles, derive from the non-segmented prechordal and pre-otic mesoderm. Recent data indicate that distinct regulatory cascades operate in the head and trunk myogenesis that rely on either Pax3 or Pax7 (Hacker and Guthrie, 1998; Mootoosamy and Dietrich, 2002). However, little is known about the normal time course and pattern of expression of these Paxgenes in relation to the expression of MRFs in postsomitic and head myogenesis. Here, we present a systematic in situ analysis of spatiotemporal characteristics of Pax3 and Pax 7 gene expression on the protein level during development of skeletal muscles of the trunk and head.

\section{Results}

Expression patterns of the Pax3, Pax7, MyoD and myogenin genes were analysed during mouse embryogenesis starting at stage E11.5 and in post-natal muscles. The respective proteins were immunohistochemically detected on adjacent paraffin sections using monoclonal antibodies, which were previously characterized as highly specific to the corresponding antigens. Expression of $M y o D$ was considered as a marker of determined myoblasts, whereas myogenin-positive cells were judged as myoblasts that are more advanced in their differentiation (see introduction). In this study, we focused on the time course and spatial expression of Pax3 and Pax7 in respect to myogenic cells identified by their expression of MRFs.

\section{Expression of Pax3 in developing muscles}

Previously it was demonstrated on the RNA level that expression of Pax3 in the trunk region starts at E8.5, coinciding with 
segmentation of the mesoderm and begins to disappear at E11.5 during somite disintegration (Goulding et al., 1991). To achieve a better understanding of the role of Pax 3 at later stages of myogenesis we have focused our analysis on the expression of Pax3 from E11.5 onwards. At E11.5 Pax3-positive cells in the somites constituted the dermomyotome and delaminating myotome. However, only cells located within the myotome expressed MyoDand myogenin(Fig. 1 A,B and data not shown). Not all cells in these structures were positive for either of these proteins. Pax3 was expressed in $79 \%( \pm 5.5 \%)$ of all cells in the MyoD-positive areas. At E12.5, staining intensity for Pax3 did not decline, but Pax3-positive cells were found in a more diffuse allocation, constituting $56 \%( \pm 4.6 \%$ ) (Fig. $1 \mathrm{C})$. This pattern of distribution of Pax3-positive cells was observed in all MyoD-positive regions, which at this stage include pre-muscle masses in different positions in the trunk and in the limbs. These muscle anlagen are known to be formed by cells actively migrating out of the myotome. In addition, Pax3 was expressed in the hypoglossal cord, consisting of myoblasts migrating from the occipital trunk somites to the tongue (Fig. 2). At E13.5, some differentiated muscle fibers can be already distinguished by the tandem locations of the myogenin-positive nuclei. Starting at this time, most of the MyoDpositive areas were Pax3-negative, including muscles of the tongue and limbs (Fig. 3 A,B and data not shown). However, in some muscle groups, e.g. those surrounding the sternum and located ventrally to the brown adipose tissue, a weak staining for Pax3 was observed in a few nuclei until E16.5 (Fig. 3C). At later stages, we did not detect Pax3-specific nuclear signals above background even after extended staining (data not shown). No Pax3 expression was detected at any stage in the MRFs-positive areas in the head (Figs. 2,4 and data not shown). In summary, the post-myotomal expression of Pax3in the trunk was characterized by a restriction in cell number and complete disappearance in most of the differentiating muscles. Furthermore, expression of
Pax3 was not detected in newborn and adult muscle tissues of the leg, trunk and head (Figs. 5,6 and data not shown). Similar results were obtained using polyclonal anti-Pax3 antibodies (data not shown).

To investigated a putative redundancy between $P a x 3$ and Pax 7 we also have analysed the expression of Pax 3 in Pax 7 knockout mice. Pax $7-/-$ embryos were examined at E11.5 and E15.5, when Pax3 is expressed and not expressed in wild type embryos, respectively. The expression of Pax3 in the mutant embryos did not differ from its expression in wild type littermates (Fig. 6 A,B and data not shown). In addition, we also did not find Pax3 protein in skeletal muscles of newborn and adult Pax7 -/mice (Fig. 6C and data not shown).

\section{Expression of Pax7 in developing muscles}

It is well known that between E9-E11 the expression of Pax7 in the trunk largely overlaps with the expression of Pax3. During somitogenesis Pax 7 is activated in the dermomyotome after Pax3 but before the onset of expression of MRFs (Jostes et al., 1990). However, little is known about expression of Pax7during myogenesis in the head. Previously, it has been reported that Pax 7 was not expressed in the head mesenchyme at E10.5 (Tajbakhsh et al., 1997). We also did not observe Pax7-positive cells in the head at this stage (data not shown). At E11.5, we detected Pax7 in cells of the MRFs-positive areas in the trunk and head mesenchyme. In the trunk, Pax7-positive cells marked the dermomyotome and myotome in a pattern very similar to Pax3 (Fig. 1A). During the next stages the spatio-temporal expression of $\mathrm{Pax} 7$ resembled that of $\mathrm{Pax} 3$. In particular, we noted a decrease of the number of the Pax7-positive cells (Fig. 1B). At E13.5, however, in contrast to Pax3, Pax7 was expressed in $35 \%$ $( \pm 3.3 \%)$ of all cells in MyoD-positive areas (Fig. 3A). At later stages, the number of Pax7-positive cells declined further (Figs. $3 \mathrm{~B}, 4$ and data not shown). However, it was difficult to quantify the
Fig. 2. Expression of $\mathrm{Pax} 3$ and $\mathrm{Pax} 7$ in the cranial region including the third branchial arch at stage E11.5. Pax3 is expressed in migrating myoblasts, which constitute the hypoglossal cord but not in the muscle anlagen of the branchial arch. In contrast to Pax3, Pax7 is expressed in the developing muscles of the branchial arch. Note, that the premuscle mass penetrated by the trigeminal nerve is in a more advanced developmental stage compared to the distal muscle mass (a Pax7 positive nucleus shown in the magnified area) and to the extrinsic eye muscles (Fig. 7) and hence contains more Pax7-positive nuclei. HC, hypoglossal cord; OP, oropharynx; TG, trigeminal (V) ganglion.

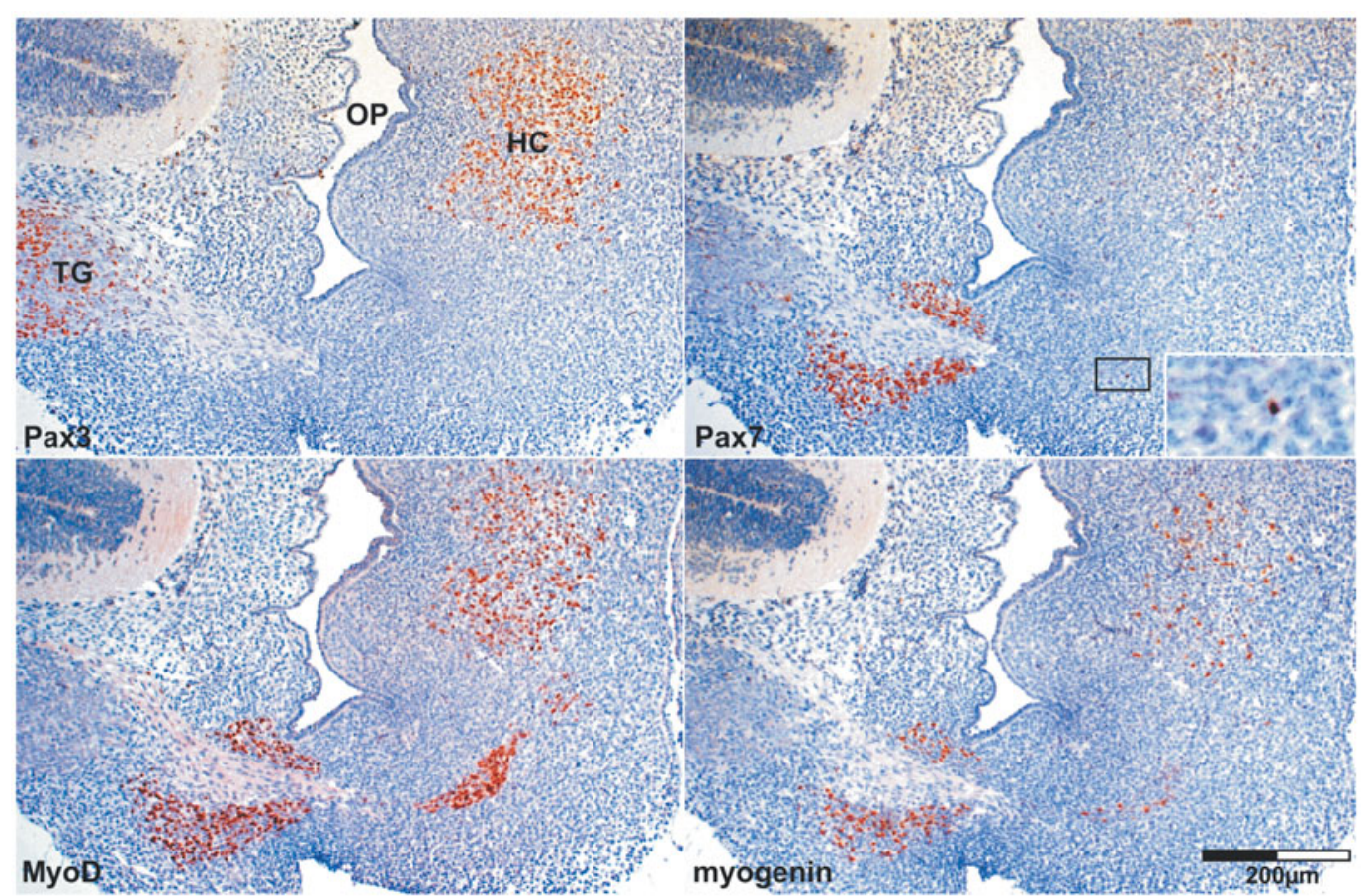


remaining Pax7-positive cells due to their irregular distribution along the differentiated myofibers, which are not fully represented on the sections. A reverse pattern of Pax 7 expression was observed during myogenesis in the head. In contrast to somites, where activity of Pax 7preceded expression of MRFs, in the head the MRFs were expressed prior to Pax7. At E11.5, MyoD and myogenin were found in small areas corresponding to the future extrinsic eye muscles (Fig. 7) and in muscle anlagen of the branchial arches, which are penetrated by nerves of the fifth cranial ganglion (Fig. 2). Myogenin was present in fewer cells than MyoD within the respective areas, reflecting a later function in myogenesis. Expression of Pax Just started at this stage and was found in a very small number of cells. Figure 7 shows that only few Pax7-positive nuclei were present on section No.2, compared to several MyoD and myogenin positive nuclei on sections No.1 and No.3, respectively. The order of sections excluded the possibility that fewer Pax7-positive nuclei were detected due to a marginal position of the section in respect to the MRFs-positive area. After E15.5, the spatial pattern of Pax7expression in the developing cranial muscles was very similar to the situation in the trunk, where Pax7 was present in only a few cells within the MyoD-positive areas (Fig. 4 and data not shown). Pax7 was found only in mononucleated cells, the number of which declined with ongoing development. Costaining with anti-laminin antibodies revealed that $\mathrm{Pax} 7$ was present exclusively in the satellite cells of head and body muscles of newborn and adult mice (Figs 5,8 and data not shown).

\section{Discussion}

\section{Pax3 is not associated with myogenic development after E13.5}

The observed expression pattern of Pax3 suggests that the function of this gene is not associated with myogenesis in the head but transiently correlates with the beginning of muscle development in the trunk and limbs. In the head only the hypoglossal cord did contain cells that were positive for MRFs and Pax3. This structure, however, does not originate from the head but is formed by myoblasts that actively migrate from the occipital trunk somites to the tongue anlage. Muscles of the developing tongue were Pax3-negative. The post-somitic expression of Pax 3 in the trunk and limbs was repressed in most muscles after E13.5. A very weak Pax3 signal, detected until E16.5 in rare nuclei of distinct muscle groups, might be explained by the different timing of the development of individual muscles. The negative staining for $\mathrm{Pax} 3$ in muscles at later stages was also corroborated using polyclonal anti-Pax3 antibodies. Since polyclonal antibodies recognize multiple epitopes, it can be excluded that the negative staining using the monoclonal antibody was due to masking of the epitope by a posttranslational modification.

Our observation that Pax3is expressed only transiently during muscle development is not in agreement with recently published data, which postulate expression of Pax3 during pre-natal and post-natal myogenesis and even in satellite cells of adult mice (Relaix et al., 2005). This apparent inconsistency might be explained by the different approaches used to detect Pax3 expression. We used a monoclonal antibody that recognizes Pax3 with a high affinity and produces no background allowing very sensitive antigen detection. The study by Relaix et al. (2005) is based on the detection of the green fluorescent protein (GFP) reporter targeted to the Pax3 gene. Obviously the artificial Pax3-GFP allele does not faithfully recapitulate expression of the endogenous Pax3 gene. It is possible that the integration of the GFP reporter gene resulted in silencing of control elements that are normally required for the down-regulation of Pax3. Alternatively, it might be envisioned that the GFP protein used to detect Pax3 expression is more stable than the actual Pax3 protein.

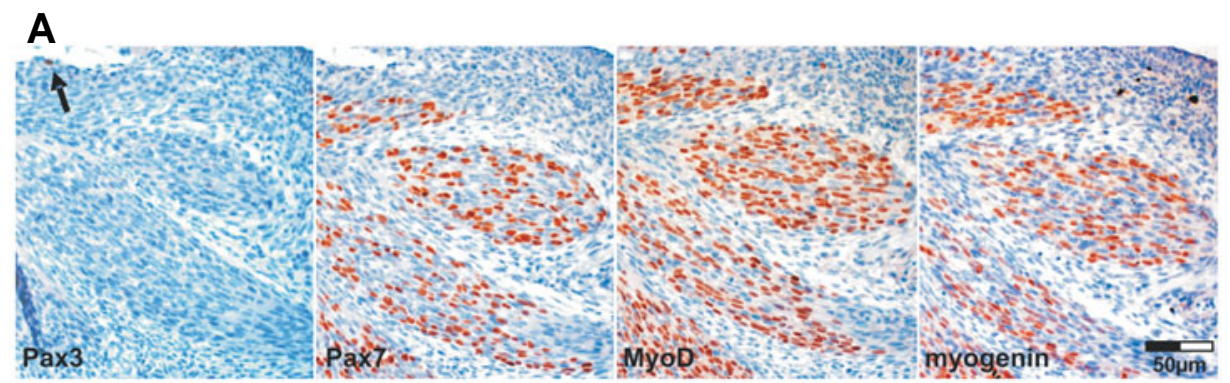

B

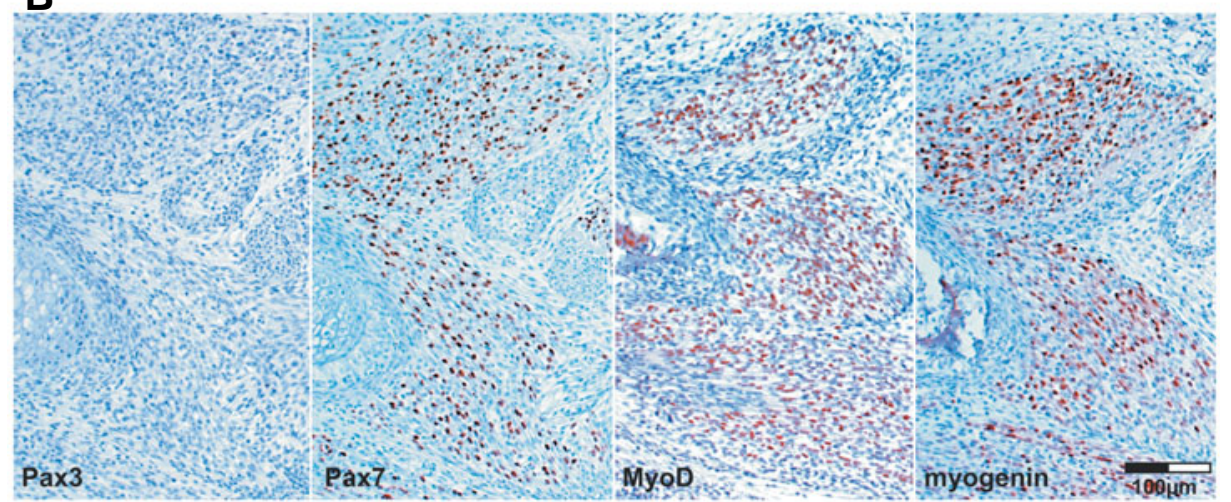

C

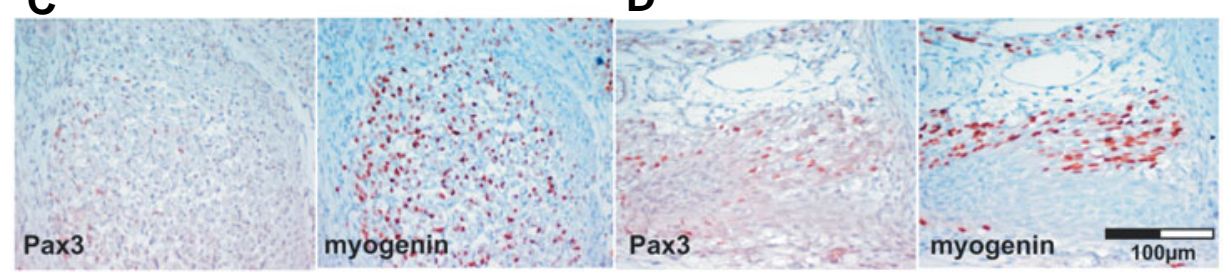

Fig. 3. Expression of Pax7 and MRFs in the developing limb muscles. (A) Stage E13.5, (B) E15.5 and (C and D) E16.5. At stage E13.5 most of the muscle groups are Pax3-negative. (A,B) represent muscle groups in a region of the humerus. (C,D) represent the Pax3-positive muscles located under the brown adipose tissue and surrounding the sternum, respectively. Note the expression of Pax 3 in melanocyte precursor cells (arrow) within the dermal layer of the skin (disrupted during sectioning), which serve as a positive control. 

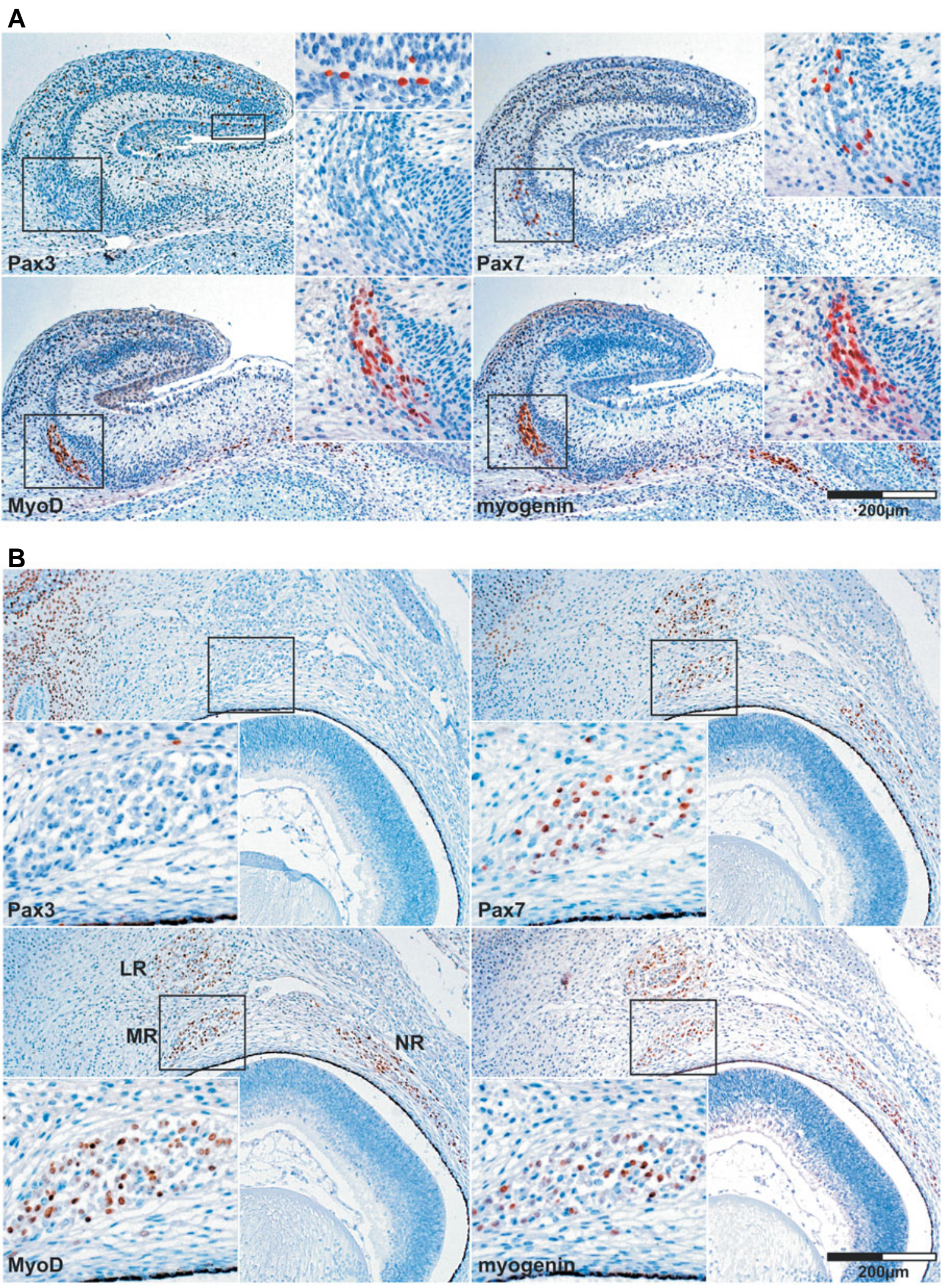

Fig. 4. Expression of Pax7 and MRFs at stage E15.5 in the developing ear (A) and extrinsic muscles of the eye (B). The muscle anlagen are Pax3 negative. LR, lateral rectus; MR, medial rectus; NR, nasal rectus. The framed areas are displayed at higher magnification. 
A

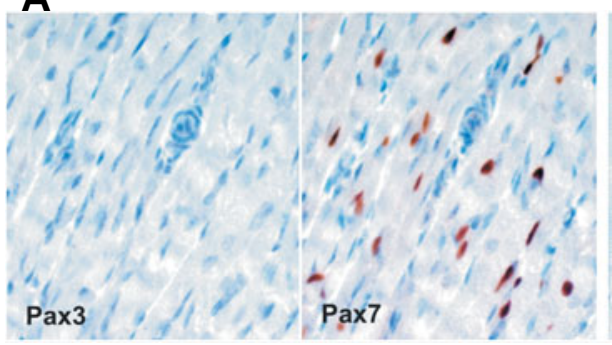

B

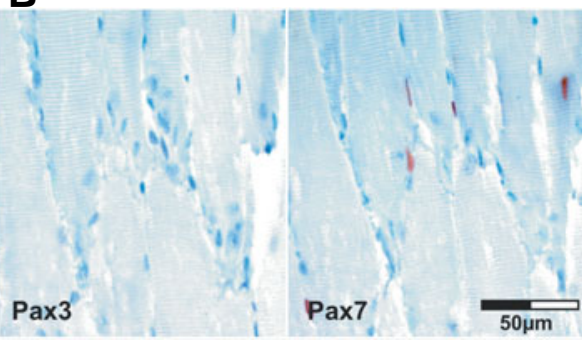

Fig. 5. Expression of Pax3 and Pax7 in the intercostal muscles of newborn (A) and adult (B) mice. Pax3 is not detected in the differentiated muscle. Pax7 is expressed in the satellite cells of both newborn and adult mice.

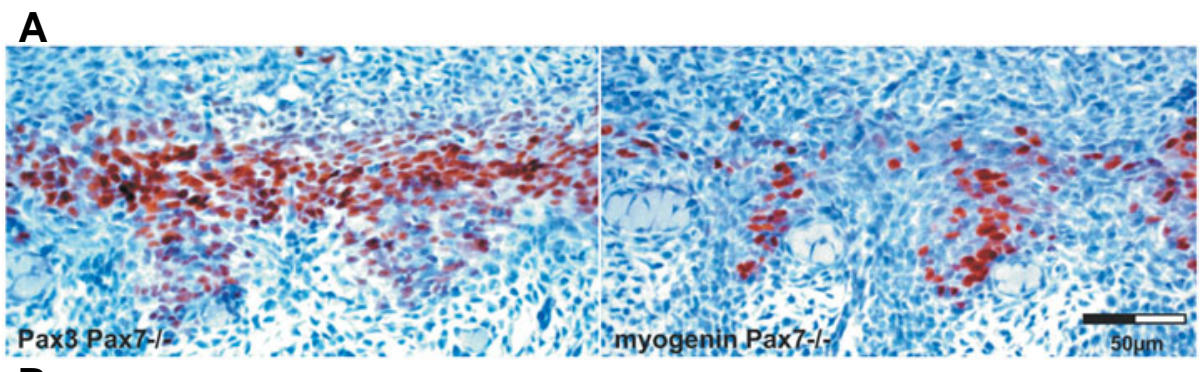

B
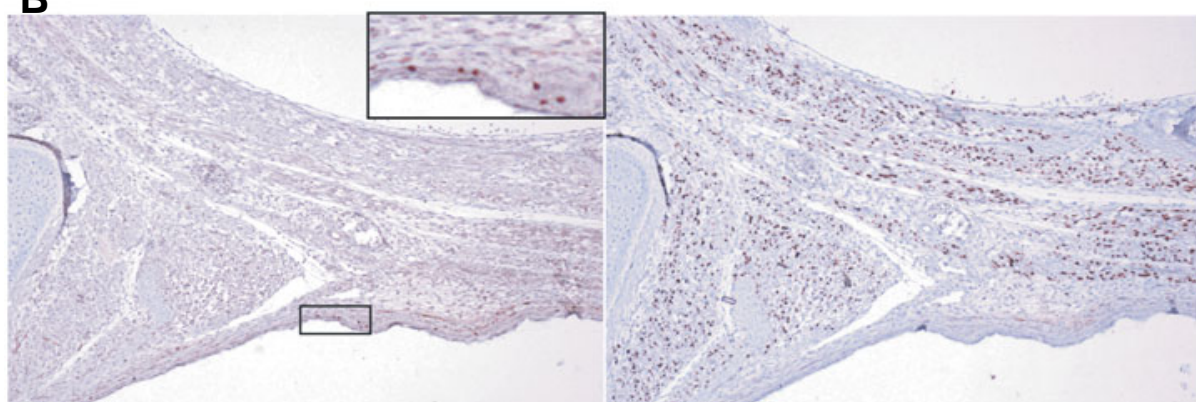

Pax3 Wt

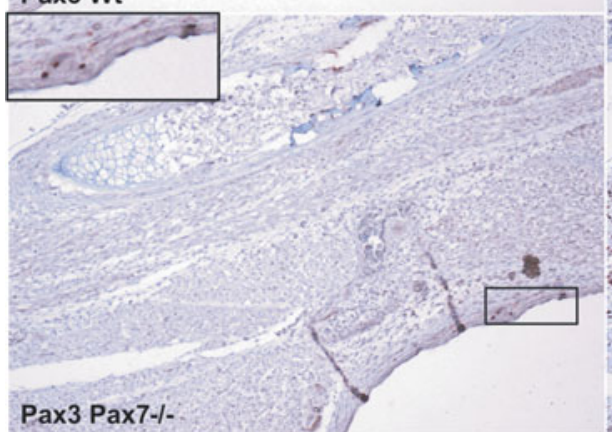

myogenin Wt

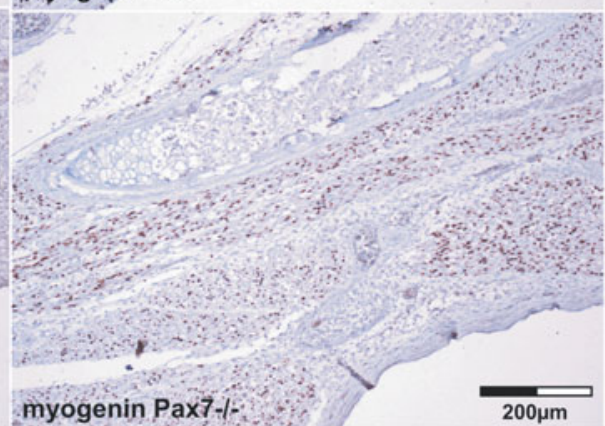

C

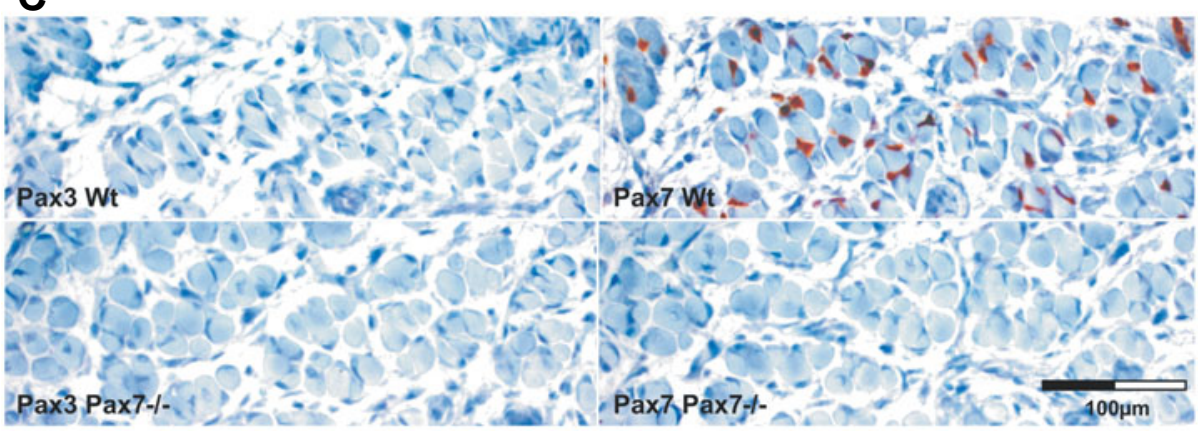

Pax3 is not de-repressed or re-activated in muscles of Pax7-/- mice

Pax3 -/- mutant embryos die at E13.5, due to multiple malformations including deformities of the heart, failure of the neural tube closure and lack those muscles, which develop from the migrating myoblast precursors (Tremblay et al., 1998). Due to the early embryo lethality it is not possible to analyse whether Pax7compensates for the absence of Pax 3 in other developing muscles. On the other hand, muscle development in Pax 7-/- mutants was unaffected (Mansouri et al., 1996). Although this finding might suggest a possible compensative role of Pax 3 we did not find a compensatory up-regulation of Pax 3 in muscles of Pax 7 mutants making this possibility less likely.

\section{Pax7 is uniformly expressed in muscles of the head and trunk}

In contrast to Pax3, Pax7is expressed in developing head muscles. Head and trunk muscles differ in their developmental origin. Muscles of the trunk derive from segmented somitic mesoderm. Cranial muscles (with the exception of the muscles of the tongue, which have a somitic origin) develop from pre-chordal and pre-otic nonsegmented mesoderm. There is experimental evidence that different regulatory cascades drive the initiation of myogenesis in the head and trunk (Hacker and Guthrie, 1998; Mootoosamy and Dietrich, 2002; Lu et al., 2002; Tzahor et al., 2003). On the other hand, a recent transplantation study established that the head environment is able to support progressive differentiation of several distinct myogenic progenitor populations from the trunk (Borue and Noden, 2004). In light of these data it was important to determine whether Pax 7 is expressed in head muscle. As shown in Figure 5, the onset of Pax 7 expression followed the expression of MRF genes suggesting that Pax Tis not directly associated with the onset of myogenesis in the head

Fig. 6. Comparison of Pax 3 expression in muscles of Pax7-/- and wild type mice. (A) Pax3 is expressed in the myotome of Pax7 mutant mice at E11.5 (see also Fig.1B). Pax3 is not de-repressed or re-activated in muscles of this mutant at E15.5 (B) and in newborns (C). Magnified areas show Pax3-positive melanocyte precursors, which serve as an internal positive control. Myogenic cells on adjacent sections are indicated by myogenin staning. $(B, C)$ represent the thoracal and hind leg muscles, respectively. 


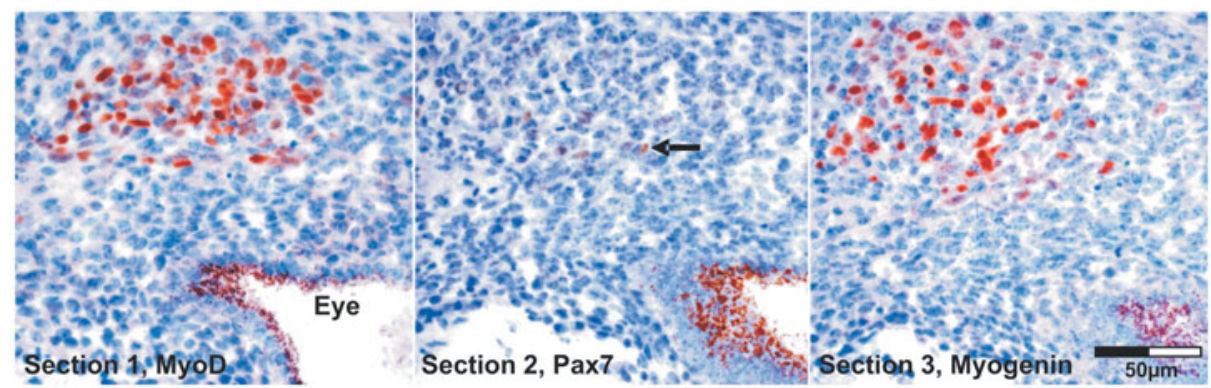

Fig. 7. Expression of Pax7 and MRFs in the lateral rectus rudiment at E11.5. Pax7 is activated later than MRFs in head muscles. Pax7 is expressed in only a few cells within the MRFs-positive area (a positive cell is shown by arrow). The order of sections ascertains that Pax 7 positive cells are located inside the MRF-positive area.

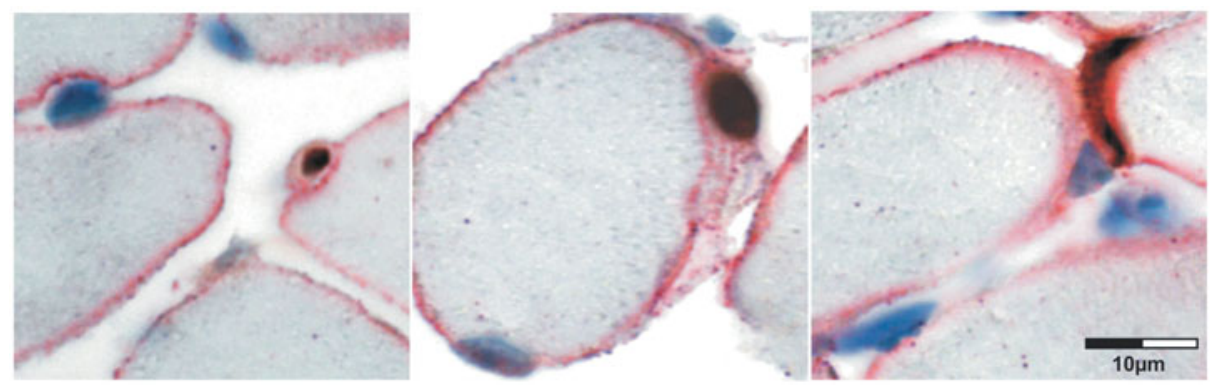

Fig. 8. Co-staining for Pax7 (brown) and laminin (red) reveals that Pax7 is expressed in the satellite cells of head muscles. Pictures represent fragments of the masseter and two facial muscles of newborn mice.

region. At later developmental stages a uniform pattern of Pax 7 expression was observed in the head and body muscles. Pax 7 was present exclusively in satellite cells suggesting a universal role in striated muscles.

\section{Pax3, Pax7 and myogenesis}

Several studies suggested a direct involvement of $P a \times 3$ and Pax 7 in the myogenic process. A direct involvement of Pax3 in myogenesis has been suggested previously by experiments based on the ectopic expression of Pax3in chicken embryos and the generation of Pax3/Myf5double knockout mice (Maroto et al., 1997; Tajbakhsh et al., 1997). The latter study demonstrated the absence of myogenesis in the trunk. However, re-examination of the original Pax3/Myf5 double knockout mice revealed that the double mutant was in fact a triple mutant, in which expression of the Mrf4 gene was additionally compromised. In fact, double knockouts of Pax3and Myf5, in which Mrf4is expressed regularly, seem to undergo normal myogenesis in the trunk (KassarDuchossoy et al., 2004). At present it is unclear whether Pax3 is directly required to activate the $M y O D$ gene in the absence of $M y f 5$ as originally postulated (Tajbakhsh et al., 1997) or whether Pax3 provides a permissive environment for the enlargement of muscle precursor cell populations, which might increase the bias for myogenic differentiation and thus enable myogenic cells to respond to environmental cues (Mennerich and Braun, 2001). Pax3 has also been shown to direct the formation of limb muscles (Bober et al., 1994). However, Pax3 is not directly linked to myogenesis in these cells. Transplantation experiments revealed that somites from Splotchembryos taken at stage E9.25 undergo muscle differentiation when grafted into the limb bud of chick host embryos (Daston et al., 1996). In double knockouts of Pax3 and Pax 7 myotomal myogenesis is initiated and essentially normal until stage E13.5 further supporting the idea that $P a x 3$ and $P a x 7$ are not required for the induction of myogenesis (Relaix et al., 2005). Our results also argue for a limited or indirect role of Pax genes for muscle development.

Striated muscles are known to exhibit identical histology and physiology in the head and trunk, suggesting that realization of the myogenic programs is similar. A main difference between head and trunk muscles, however, is the mode of induction. Here, we have demonstrated that neither Pax7nor Pax3are likely to serve a role in the initiation of myogenesis in head muscles: (i) Pax3is not expressed in head muscles; (ii) head muscle development is not affected in Pax7--/- and splotch mice; (iii) Pax 7 is expressed only after expression of MRFs in head muscles. Moreover, Pax3is not de-repressed in Pax7-/- mice, in which muscle development proceeds normally, suggesting that after E13.5 myogenesis does not require $P a x 3$ and Pax7. Taken together it seems not likely that the core program of striated muscle development depends on Paxgenes. Given the relatively short period of Pax3 expression in the trunk until stage E13.5 (in some muscles - until E16.5) it seems likely that the Pax3/7-cell population of muscle progenitors (Relaix et al., 2005) exists only transiently and does not constitute a persistent cell lineage.

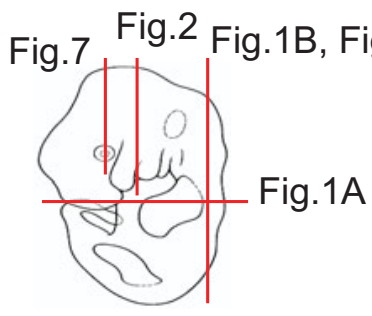

E 11.5

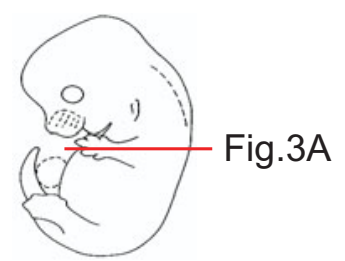

E 13.5
Fig. 1C

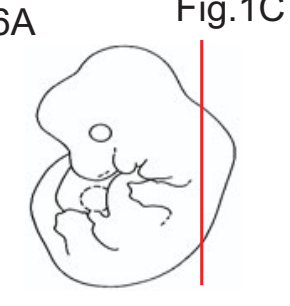

E 12.5

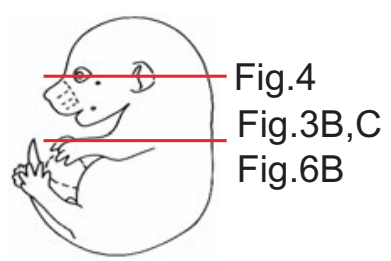

E 15.5 and E16.5
Fig. 9. Schematic representation of embryos and positions of the sections shown in the respective figures. 
Based on these arguments it is tempting to speculate that $P$ ax 3 and Pax7allow the formation of provisory myogenic structures, in which the myogenic process itself is initiated as a distinct program independent of Pax genes. Further experiments are required to reveal specific instructive roles of Pax3and Pax7for myogenesis.

\section{Materials and Methods}

\section{Genotyping of the Pax7 knockout embryos}

The generation of the Pax $7-L a c Z$ mutant mice has been published (Mansouri et al., 1996). The mutant mice were maintained on a mixed $129 \mathrm{~Sv} / \mathrm{C} 57 / \mathrm{BI} 6$ background. Embryos were genotyped by PCR and Southern blot analysis, the absence of Pax7 protein was also corroborated by immunohistochemical stainings.

\section{Immunohistochemistry}

Embryos of wild type Balb/c mice were fixed in $4 \%$ paraformaldehyde and embedded into paraffin according to standard protocols. Adjacent 7 $\mu \mathrm{m}$ sections were used for comparative analysis. Endogenous peroxidase was inactivated in $0.3 \% \mathrm{H}_{2} \mathrm{O}_{2}$ in methanol. For antigen unmasking, the slides were autoclaved in $1 \mathrm{mM}$ citrate buffer, $\mathrm{pH}$ 6.0. Immunodetection was performed with the primary mouse monoclonal antibodies anti-MyoD (DakoCytomation, Clone 5.8A), anti-myogenin (DakoCytomation, Clone F5D), anti-Pax3 developed by C. P. Ordahl and anti-Pax7 developed by A. Kawakami. The Pax3 and Pax7 antibodies were obtained from the Developmental Studies Hybridoma Bank (NICHD; The University of lowa, Department of Biological Sciences, lowa City, IA 52242). Rabbit polyclonal anti-Pax3 antibody was kindly provided by J. Epstein, University of Pennsylvania, Philadelphia, PA. The antibodies were applied with the following dilutions: 1:50 for anti-MyoD, 1:100 for anti-myogenin, 1:1000 for anti-Pax3 and anti-Pax7, 1:1000 for pre-absorbed polyclonal antiPax3. For detection of primary antibodies we used biotinylated rabbitanti-mouse antibody (DakoCytomation) and the avidin-biotin-complex (ABC, Vector). Anti-laminin rabbit polyclonal antibodies (L-9393, Sigma) were applied (1:200) after an additional unmasking treatment with proteinase $\mathrm{K}$ (Sigma), $100 \mu \mathrm{g} / \mathrm{ml}$, for $8 \mathrm{~min}$ at $37^{\circ} \mathrm{C}$. The anti-laminin antibodies were detected by donkey anti-rabbit-alkaline phosphatase conjugates (Dianova) and subsequent staining with the Fast Red alkaline phosphatase substrate (Sigma). Finally, the sections were counterstained with hematoxylin (DakoCytomation).

\section{Cell quantification}

Two embryos per stage were analysed. Cell counting was achieved using the Adobe Photoshop program. On a high magnification image of a selected area, positively stained nuclei were marked by dots and counted. The counting results were tested for consistency using a ChiSquare test $(n=12, p \alpha=0.01)$. The differences between $E 11.5$ and E12.5 and between E12.5 and E13.5 were shown to be significant ( $p$-Test of binomial distributions; $p \alpha=0.01$ ), mean values were taken.

\section{Acknowledgements}

We thank Jonathan A. Epstein for providing the polyclonal anti-Pax3 antibodies, Anita Brandstätter for statistical data evaluation, Petra Pennekamp and Konstantinos Bilbilis for helpful discussions. This study was supported by the Deutsche Forschungsgemeinschaft.

\section{References}

ARNOLD, H.H. and BRAUN, T. (1996). Targeted inactivation of myogenic factor genes reveals their role during mouse myogenesis: a review. Int. J. Dev. Biol. 40: 345-353.

BOBER, E., FRANZ, T., ARNOLD, H.H., GRUSS, P., TREMBLAY, P. (1994). Pax-3 is required for the development of limb muscles: a possible role for the migration of dermomyotomal muscle progenitor cells. Development 120: 603-612.
BORUE, X., NODEN D.M. (2004). Normal and aberrant craniofacial myogenesis by grafted trunk somitic and segmental plate mesoderm. Development131: 39673980.

DASTON, G., LAMAR, E., OLIVIER, M., GOULDING, M. (1996). Pax-3 is necessary for migration but not differentiation of limb muscle precursors in the mouse. Development 122: 1017-1027.

GOULDING, M.D., CHALEPAKIS, G., DEUTSCH, U., ERSELIUS, J.R., GRUSS, P. (1991). Pax-3, a novel murine DNA binding protein expressed during early neurogenesis. EMBO J. 10: 1135-1147.

GOULDING, M., LUMSDEN, A., PAQUETTE, A.J. (1994). Regulation of Pax-3 expression in the dermomyotome and its role in muscle development. Development 120: 957-971.

HACKER, A., GUTHRIE, S. (1998). A distinct developmental programme for the cranial paraxial mesoderm in the chick embryo. Development 125: 3461-3472.

HASTY, P., BRADLEY, A., MORRIS, J.H., EDMONDSON, D.G., VENUTI, J.M., OLSON, E.N., KLEIN, W.H. (1993). Muscle deficiency and neonatal death in mice with a targeted mutation in the myogenin gene. Nature 364: 501-506.

JOSTES, B., WALTHER, C., GRUSS, P. (1990). The murine paired box gene, Pax7, is expressed specifically during the development of the nervous and muscular system. Mech. Dev. 33: 27-37.

KASSAR-DUCHOSSOY, L., GAYRAUD-MOREL, B., GOMES, D., ROCANCOURT, D., BUCKINGHAM, M., SHININ, V., TAJBAKHSH, S. (2004). Mrf4 determines skeletal muscle identity in Myf5:Myod double-mutant mice. Nature 431: 466471.

LU, J.R., BASSEL-DUBY, R., HAWKINS, A., CHANG, P., VALDEZ, R., WU, H., GAN, L., SHELTON, J.M., RICHARDSON, J.A., OLSON, E.N. (2002). Control of facial muscle development by MyoR and capsulin. Science 298: 2378-2381.

MANSOURI, A., STOYKOVA, A., TORRES, M., GRUSS, P. (1996). Dysgenesis of cephalic neural crest derivatives in Pax7-/- mutant mice. Development 122: 831-838.

MAROTO, M., RESHEF, R., MUNSTERBERG, A.E., KOESTER, S., GOULDING, M., LASSAR, A.B. (1997). Ectopic Pax-3 activates MyoD and Myf-5 expression in embryonic mesoderm and neural tissue. Cel/89: 139-148.

MENNERICH, D., BRAUN, T. (2001). Activation of myogenesis by the homeobox gene Lbx1 requires cell proliferation. EMBO $J$ 20: 7174-7183.

MOOTOOSAMY, R.C., DIETRICH, S. (2002). Distinct regulatory cascades for head and trunk myogenesis. Development 129: 573-583.

NABESHIMA, Y., HANAOKA, K., HAYASAKA, M., ESUMI, E., LI, S., NONAKA, I., NABESHIMA, Y. (1993). myogenin gene disruption results in perinatal lethality because of severe muscle defect. Nature 364: 532-535.

OUSTANINA, S., HAUSE, G., BRAUN, T. (2004). Pax7 directs postnatal renewal and propagation of myogenic satellite cells but not their specification. EMBOJ. 23: 3430-3439.

RELAIX F, ROCANCOURT D, MANSOURI A, BUCKINGHAM M. (2005). A Pax3/ Pax7-dependent population of skeletal muscle progenitor cells. Nature. 435: 948-953.

RUDNICKI MA, SCHNEGELSBERG PN, STEAD RH, BRAUN T, ARNOLD HH, JAENISCH R. MyoD or Myf-5 is required for the formation of skeletal muscle. Cell. (1993) 75: 1351-1359.

SEALE, P., SABOURIN, L.A., GIRGIS-GABARDO, A., MANSOURI, A., GRUSS, P., RUDNICKI, M.A. (2000). Pax7is required for the specification of myogenic satellite cells. Cel/102: 777-786.

TAJBAKHSH, S., ROCANCOURT, D., COSSU, G., BUCKINGHAM, M. (1997). Redefining the genetic hierarchies controlling skeletal myogenesis: Pax-3 and Myf-5 act upstream of MyoD. Cel/ 89: 127-138.

TREMBLAY, P., DIETRICH, S., MERICSKAY, M., SCHUBERT, F.R., LI, Z., PAULIN, D. (1998). A crucial role for Pax3 in the development of the hypaxial musculature and the long-range migration of muscle precursors. Dev. Biol. 203: 49-61.

TZAHOR, E., KEMPF, H., MOOTOOSAMY, R.C., POON, A.C., ABZHANOV, A., TABIN, C.J., DIETRICH, S., LASSAR, A.B. (2003). Antagonists of Wnt and BMP signaling promote the formation of vertebrate head muscle. Genes Dev. 17: 3087-3099.

Received: August 2005

Reviewed by Referees: September 2005

Modified by Authors and Accepted for Publication: October 2005

Edited by: Christine Mummery 